Analele Universităţii de Vest, Timişoara

Seria Matematică - Informatică

LIII, 2, (2015), 17- 28

\title{
Integral inequalities for (Orlicz, Breckner) co-ordinated convex functions on rectangles
}

Gabriela Cristescu, Muhammad Aslam Noor, Muhammad Uzair Awan, and Mihail Găianu

\begin{abstract}
The concept of (Orlicz, Breckner) co-ordinated $(s, \sigma)$ convexity is introduced for two variables real functions. Upper and lower sharp integral boundary properties of Hermite-Hadamard type are proved on rectangles.
\end{abstract}

AMS Subject Classification (2000). 26D15 ; 26A51

Keywords. convex function, Breckner-Orlicz convex function on coordinates, Hermite-Hadamard type inequality

\section{Introduction}

Let us denote by $\mathbb{R}, \mathbb{Q}, \mathbb{Z}$ and $\mathbb{N}$, respectively, the set of all real, rational, integer and natural numbers. Let us consider the numbers $a, b, c, d \in[0,+\infty)$ such as $a<b$ and $c<d$, and a rectangle $D=[a, b] \times[c, d]$. By $L_{1}(D)$ we denote the set of all Lebesque integrable functions over the rectangle $D$. It is well known that a function $f:[a, b] \rightarrow \mathbb{R}$ is convex on $[a, b]$ if and only if the following inequality

$$
f\left(\frac{p+q}{2}\right) \leq \frac{1}{p-q} \int_{p}^{q} f(x) d x \leq \frac{f(p)+f(q)}{2},
$$

holds whenever $p, q \in[a, b]$ and $p<q$. The reverse inequality characterizes the concave functions. The inequality is sharp for affine functions. 
The concept of convex function (classical convexity [15]), was extended in many directions and frameworks due to its numerous applications in optimization, variational methods, geometry and artificial intelligence. Moving to functions defined on spaces with more dimensions is one of the directions of generalization. The first Hermite-Hadamard type inequalities for co-ordinated convex functions in two dimensions are in [11]. Various kinds of generalized convexity are extended to functions of several variables and generated integral inequalities of Hermite-Hadamard type (see, for example, $[1],[4],[18])$.

The properties of $s$-convexity generate the framework of this paper. Let $s \in(0,1]$. The first concept of $s$-convexity was defined in 1961 by W. Orlicz $[20]:$

Definition 1.1. A function $f:[0,+\infty) \rightarrow \mathbb{R}$ is said to be $s$-convex in the first sense (or Orlicz-convex) if

$$
f(\alpha x+\beta y) \leq \alpha^{s} f(x)+\beta^{s} f(y),
$$

for all $x, y \in[0,+\infty)$ and $\alpha, \beta \in[0,1]$ with $\alpha^{s}+\beta^{s}=1$. If the inequality is strict then $f$ is said to be strictly s-convex in the first sense. The reverse inequality defines the s-concavity in the first sense (or Orlicz concavity) of $f$. If the inequality is sharp then $f$ is called s-affine in the first sense.

A later $s$-convexity concept is in the following definition, given in 1978 by W. Breckner [5]:

Definition 1.2. A function $f:[0,+\infty) \rightarrow \mathbb{R}$ is said to be s-convex in the second sense (or Breckner-convex) if

$$
f(t x+(1-t) y) \leq t^{s} f(x)+(1-t)^{s} f(y)
$$

for all $x, y \in[0,+\infty)$ and $t \in[0,1]$. If the inequality is strict then $f$ is said to be strictly s-convex in the second sense. The reverse inequality defines the $s$-concavity in the second sense (or Breckner concavity) of $f$. If the inequality is sharp then $f$ is called s-affine in the second sense.

The main properties of the functions having either Breckner or Orlicz convexity properties are in [6], [10], [14]. The relationship between the two $s$-convexity properties is studied in [10] and [14]. Integral inequalities of Hermite-Hadamard type within various classes of $s$-convex functions are numerous, depending on particular additional properties (see, for example, [7], [8], [9], [10], [19]). 
In this paper we intend to derive some integral inequalities of HermiteHadamard type within classes of two variables functions having Orlicz and Breckner convexities on co-ordinates. We consider functions $f:[0,+\infty)^{2} \rightarrow$ $\mathbb{R}$, restricting the research to particular rectangles $D$. Define, as usual, the partial mappings $f_{y}:[0,+\infty) \rightarrow \mathbb{R}, f_{y}(u)=f(u, y)$ and $f_{x}:[0,+\infty) \rightarrow \mathbb{R}$, $f_{x}(v)=f(x, v)$. Let us chose the fixed numbers $s, \sigma \in(0,1]$.

Definition 1.3. A function $f:[0,+\infty)^{2} \rightarrow \mathbb{R}$ is said to be:

1. Breckner co-ordinated $(s, \sigma)$-convex on $D$ if the partial function $f_{y}$ is $s$-convex in the second sense for all $y \in[c, d]$ and $f_{x}$ is $\sigma$-convex in the second sense for all $x \in[a, b]$.

2. (Orlicz, Breckner) co-ordinated $(s, \sigma)$-convex on $D$ if the partial function $f_{y}$ is s-convex in the first sense for all $y \in[c, d]$ and $f_{x}$ is $\sigma$-convex in the second sense for all $x \in[a, b]$.

3. Orlicz co-ordinated $(s, \sigma)$-convex on $D$ if the partial function $f_{y}$ is $s$ convex in the first sense for all $y \in[c, d]$ and $f_{x}$ is $\sigma$-convex in the first sense for all $x \in[a, b]$.

The corresponding concepts of co-ordinated concavity are defined by reversing the properties of the partial mappings. Also, the corresponding concept of affine function on one or both co-ordinates are defined by means of the corresponding properties of the partial mappings.

Corollary 1.1. A functionf $:[0,+\infty)^{2} \rightarrow \mathbb{R}$ is

1. Breckner co-ordinated $(s, \sigma)$-convex on $D$ if and only if

$$
\begin{gathered}
f\left(\alpha x_{1}+\beta x_{2}, \gamma y_{1}+\delta y_{2}\right) \\
\leq \alpha^{s} \gamma^{\sigma} f(a, c)+\alpha^{s} \delta^{\sigma} f(a, d)+\beta^{s} \gamma^{\sigma} f(b, c)+\beta^{s} \delta^{\sigma} f(b, d),
\end{gathered}
$$

for all $\left(x_{1}, y_{1}\right),\left(x_{2}, y_{2}\right) \in D$ and $\alpha, \beta, \gamma, \delta \in[0,1]$ with $\alpha+\beta=1$ and $\gamma+\delta=1$.

2. (Orlicz, Breckner) co-ordinated $(s, \sigma)$-convex on $D$ if and only if (1.4) holds for all $\left(x_{1}, y_{1}\right),\left(x_{2}, y_{2}\right) \in D$ and $\alpha, \beta, \gamma, \delta \in[0,1]$ with $\alpha^{s}+\beta^{s}=1$ and $\gamma+\delta=1$.

3. Orlicz co-ordinated $(s, \sigma)$-convex on $D$ if and only if (1.4) holds for all $\left(x_{1}, y_{1}\right),\left(x_{2}, y_{2}\right) \in D$ and $\alpha, \beta, \gamma, \delta \in[0,1]$ with $\alpha^{s}+\beta^{s}=1$ and $\gamma^{\sigma}+\delta^{\sigma}=1$. 
The Breckner co-ordinated $(s, \sigma)$-convex functions are frequent in the inequalities literature. Many techniques of approach generated inequalities of Hermite-Hadamard type for this kind of functions (see [2], [3], [21]). Additional properties of functions, such as differentiability, generate many integral inequalities as in [16], [23], [24]. The Orlicz co-ordinated $(s, \sigma)$-convexity is less approached from the point of view of integral inequalities [22]. The properties of convexity of the partial mappings are identic in the most part of the literature referring to the integral inequalities (see [1], [11]). Many times the partial mappings are $s$-convex in the second sense with the same $s([2],[3],[16],[21])$ or with distinct values $s_{1}$ and $s_{2}$, as in [4]. The Orlicz convexity of the partial mappings, by means of two distinct values $s_{1}$ and $s_{2}$ is approached in [22] . As consequence, there are Hermite-Hadamard type inequalities both in case of the Breckner co-ordinated $(s, \sigma)$-convexity and of the Orlicz co-ordinated $(s, \sigma)$-convexity.

There is a class of functions, for which the two $s$-convexities coincide, which was approached in the previously quoted papers. We deal with the case of those functions that have distinct convexities, not implying each-other. In this paper we derive new Hermite-Hadamard type inequalities the class of (Orlicz, Breckner) co-ordinated $(s, \sigma)$-convex functions on rectangles, which combines two convexities.

\section{Lower bounds of Hermite-Hadamard type}

All the two variables functions considered in this section have (Orlicz, Breckner) co-ordinated convexity property on a rectangle $D \subseteq[0,+\infty)$ and are Lebesque integrable on $D$. The properties of the Orlicz-convex functions (see [14]) and of the Breckner-convex functions (see [6]) show that this class of functions is very numerous.

Lemma 2.1. Let two numbers $s, \sigma \in(0,1]$, not necessarily distinct, be fixed. Let $f:[0,+\infty)^{2} \rightarrow \mathbb{R}$ be a function that is (Orlicz, Breckner) co-ordinated $(s, \sigma)$-convex on its domain. Then

$$
\begin{gathered}
f\left(\frac{x_{1}+x_{2}}{2^{\frac{1}{s}}}, \frac{y_{1}+y_{2}}{2}\right) \\
\leq \frac{1}{2^{\sigma+1}}\left[f\left(x_{1}, y_{1}\right)+f\left(x_{1}, y_{2}\right)+f\left(x_{2}, y_{1}\right)+f\left(x_{2}, y_{2}\right)\right],
\end{gathered}
$$

for all $\left(x_{1}, y_{1}\right),\left(x_{2}, y_{2}\right) \in[0,+\infty)^{2}$. 
Proof. Since $f$ is (Orlicz, Breckner) co-ordinated $(s, \sigma)$-convex, we use Corollary 1.1 taking $\alpha=t, \beta=\left(1-t^{s}\right)^{\frac{1}{s}}, \gamma=\lambda$ and $\delta=1-\lambda, t, \lambda \in[0,1]$. Then (1.4) becomes

$$
\begin{gathered}
f\left(t x_{1}+\left(1-t^{s}\right)^{\frac{1}{s}} x_{2}, \lambda y_{1}+(1-\lambda) y_{2}\right) \\
\leq t^{s} \lambda^{\sigma} f\left(x_{1}, y_{1}\right)+t^{s}(1-\lambda)^{\sigma} f\left(x_{1}, y_{2}\right) \\
+\left(1-t^{s}\right) \lambda^{\sigma} f\left(x_{2}, y_{1}\right)+\left(1-t^{s}\right)(1-\lambda)^{\sigma} f\left(x_{2}, y_{2}\right) .
\end{gathered}
$$

Taking $t=\frac{1}{2^{\frac{1}{s}}}$ and $\lambda=\frac{1}{2}$ in this inequality, one gets the required result.

Theorem 2.2. Let two numbers $s, \sigma \in(0,1]$, not necessarily distinct, be fixed. Let $f:[0,+\infty)^{2} \rightarrow \mathbb{R}$ be a function that is (Orlicz, Breckner) coordinated $(s, \sigma)$-convex and $f \in L_{1}(D)$ on each rectangle $D=[a, b] \times[c, d] \subseteq$ $[0,+\infty)$. Then

$$
\begin{gathered}
f\left(\frac{a+b}{2^{\frac{1}{s}}}, \frac{c+d}{2}\right) \\
\leq \frac{1}{2^{\sigma}(b-a)^{s}(d-c)} \iint_{(D)} f(x, y)\left[(b-x)^{s-1}+(x-a)^{s-1}\right] d x d y .
\end{gathered}
$$

Proof. In the inequality from Lemma 2.1 one takes $x_{1}, x_{2} \in[a, b]$ and $y_{1}, y_{2} \in$ $[c, d]$ expressed as convex combinations of the interval extremities, as follows: $x_{1}=t^{\frac{1}{s}} a+\left(1-t^{\frac{1}{s}}\right) b, x_{2}=\left(1-t^{\frac{1}{s}}\right) a+t^{\frac{1}{s}} b, y_{1}=\tau c+(1-\tau) d, y_{2}=(1-\tau) c+\tau d$, with $t, \tau \in[0,1]$. Then $x_{1}+x_{2}=a+b$ and $y_{1}+y_{2}=c+d$ and one gets:

$$
\begin{gathered}
2^{\sigma+1} f\left(\frac{a+b}{2^{\frac{1}{s}}}, \frac{c+d}{2}\right) \\
\leq f\left(t^{\frac{1}{s}} a+\left(1-t^{\frac{1}{s}}\right) b, \tau c+(1-\tau) d\right)+f\left(t^{\frac{1}{s}} a+\left(1-t^{\frac{1}{s}}\right) b,(1-\tau) c+\tau d\right) \\
+f\left(\left(1-t^{\frac{1}{s}}\right) a+t^{\frac{1}{s}} b, \tau c+(1-\tau) d\right)+f\left(\left(1-t^{\frac{1}{s}}\right) a+t^{\frac{1}{s}} b,(1-\tau) c+\tau d\right) .
\end{gathered}
$$

Since $f \in L_{1}(D)$ we can double integrate this inequality with respect to $t$ over $[0,1]$ and with respect to $\tau$ over $[0,1]$. One gets:

$$
\begin{gathered}
2^{\sigma+1} f\left(\frac{a+b}{2^{\frac{1}{s}}}, \frac{c+d}{2}\right) \\
\leq \int_{0}^{1} \int_{0}^{1} f\left(t^{\frac{1}{s}} a+\left(1-t^{\frac{1}{s}}\right) b, \tau c+(1-\tau) d\right) d t d \tau \\
+\int_{0}^{1} \int_{0}^{1} f\left(t^{\frac{1}{s}} a+\left(1-t^{\frac{1}{s}}\right) b,(1-\tau) c+\tau d\right) d t d \tau \\
+\int_{0}^{1} \int_{0}^{1} f\left(\left(1-t^{\frac{1}{s}}\right) a+t^{\frac{1}{s}} b, \tau c+(1-\tau) d\right) d t d \tau
\end{gathered}
$$




$$
+\int_{0}^{1} \int_{0}^{1} f\left(\left(1-t^{\frac{1}{s}}\right) a+t^{\frac{1}{s}} b,(1-\tau) c+\tau d\right) d t d \tau .
$$

Let us change the variables as follows:

- Put $t^{\frac{1}{s}} a+\left(1-t^{\frac{1}{s}}\right) b=x$ in the first two integrals. It means that $d t=\frac{-s}{(b-a)^{s}}(b-x)^{s-1} d x, x(0)=b, x(1)=a$;

- Put $\left(1-t^{\frac{1}{s}}\right) a+t^{\frac{1}{s}} b=x$ in the third and the fourth integrals. It means that $d t=\frac{s}{(b-a)^{s}}(x-a)^{s-1} d x, x(0)=a, x(1)=b$;

- Put $\tau c+(1-\tau) d=y$ in the first and the third integrals. It means that $d \tau=\frac{-1}{d-c} d y, y(0)=d, y(1)=c$;

- Put $(1-\tau) c+\tau d=y$ in the second and the fourth integrals. It means that $d \tau=\frac{1}{d-c} d y, y(0)=c, y(1)=d$.

The previous inequality becomes:

$$
\begin{gathered}
f\left(\frac{a+b}{2^{\frac{1}{s}}}, \frac{c+d}{2}\right) \\
\leq \frac{2 s}{2^{\sigma+1}(b-a)^{s}(d-c)} \int_{a}^{b} \int_{c}^{d} f(x, y)(b-x)^{s-1} d x d y \\
+\frac{2 s}{2^{\sigma+1}(b-a)^{s}(d-c)} \int_{a}^{b} \int_{c}^{d} f(x, y)(x-a)^{s-1} d x d y,
\end{gathered}
$$

which yields the required inequality.

Theorem 2.3. Let two numbers $s, \sigma \in(0,1]$, not necessarily distinct, be fixed. Let $f:[0,+\infty)^{2} \rightarrow \mathbb{R}$ be a function that is (Orlicz, Breckner) coordinated $(s, \sigma)$-convex and $f \in L_{1}(D)$ on each rectangle $D=[a, b] \times[c, d] \subseteq$ $[0,+\infty)$. Then

$$
f\left(\frac{a+b}{2^{\frac{1}{s}}}, \frac{c+d}{2}\right) \leq \frac{1}{2^{\sigma-1}(b-a)(d-c)} \iint_{(D)} f(x, y) d x d y .
$$

Proof. The proof follows the same steps as the proof of the previous theorem. Except that we make the following change of variables in inequality (2.3):

- Put $t a+(1-t) b=x$ in the first two integrals.

- Put $(1-t) a+t b=x$ in the third and the fourth integrals.

- Substitute the second coordinate in the same manner as in the proof of the previous theorem. 
The inequality (2.3) becomes

$$
f\left(\frac{a+b}{2^{\frac{1}{s}}}, \frac{c+d}{2}\right) \leq \frac{4}{2^{\sigma+1}} \iint_{(D)} f(x, y) \frac{1}{(b-a)(d-c)} d x d y
$$

which yields the expected result.

Remark 2.1. Both inequality (2.2) and (2.4) are sharp. To prove their sharpness we chose function $f$ to be Orlicz-affine in the first co-ordinate and Breckner-affine in the second co-ordinate. Both kinds of affine functions are constant, according to [17] Corollary 3.3., for all numbers $s$ and $\sigma$. Let us take $s=\sigma=1$ and $f(x, y)=k$ constant. Elementary calculus shows that both inequalities become $k \leq k$ in this case, which shows their sharpness.

Remark 2.2. The inequalities (2.2) and (2.4) are identic if $s=1$, which means in case of functions that are convex (in classical sense) with respect to the first co-ordinate.

Remark 2.3. If $s=\sigma=1$ then both inequalities (2.2) and (2.4) reduce to the lower bound from [11], Theorem 1.

\section{$3 \quad$ Upper bounds of Hermite-Hadamard type}

Theorem 3.1. Let two numbers $s, \sigma \in(0,1]$, not necessarily distinct, be fixed. Let $f:[0,+\infty)^{2} \rightarrow \mathbb{R}$ be a function that is (Orlicz, Breckner) coordinated $(s, \sigma)$-convex and $f \in L_{1}(D)$ on each rectangle $D=[a, b] \times[c, d] \subseteq$ $[0,+\infty)$. Then

$$
\begin{gathered}
\int_{c}^{d} \int_{0}^{1} f\left(t a+\left(1-t^{s}\right)^{\frac{1}{s}} b, y\right)\left[1+t^{s-1}\left(1-t^{s}\right)^{\frac{1-s}{s}}\right] d t d y \\
\leq \frac{d-c}{\sigma+1}[f(a, c)+f(a, d)+f(b, c)+f(b, d)] .
\end{gathered}
$$

Proof. The $s$-convexity in the first sense of function $f$ with respect to the first coordinate means that taking $t \in[0,1]$ and $y \in[c, d]$ one gets the following inequalities:

$$
\begin{aligned}
& f\left(t a+\left(1-t^{s}\right)^{\frac{1}{s}} b, y\right) \leq t^{s} f(a, y)+\left(1-t^{s}\right) f(b, y), \\
& f\left(\left(1-t^{s}\right)^{\frac{1}{s}} a+t b, y\right) \leq\left(1-t^{s}\right) f(a, y)+t^{s} f(b, y) .
\end{aligned}
$$


Compute the sum of (3.2) and (3.3) and get

$$
f\left(t a+\left(1-t^{s}\right)^{\frac{1}{s}} b, y\right)+f\left(\left(1-t^{s}\right)^{\frac{1}{s}} a+t b, y\right) \leq f(a, y)+f(b, y) .
$$

Integrate this inequality with respect to $t$ over $[0,1]$.

$$
\begin{gathered}
\int_{0}^{1} f\left(t a+\left(1-t^{s}\right)^{\frac{1}{s}} b, y\right) d t+\int_{0}^{1} f\left(\left(1-t^{s}\right)^{\frac{1}{s}} a+t b, y\right) d t \\
\leq f(a, y)+f(b, y) .
\end{gathered}
$$

Substitute $p=\left(1-t^{s}\right)^{\frac{1}{s}}$ in the second integral. It means that

$$
d t=-p^{s-1}\left(1-p^{s}\right)^{\frac{1-s}{s}} d p,
$$

$p(0)=1$ and $p(1)=0$. The inequality (3.4) becomes

$$
\begin{gathered}
\int_{0}^{1} f\left(t a+\left(1-t^{s}\right)^{\frac{1}{s}} b, y\right) d t \\
+\int_{0}^{1} f\left(p a+\left(1-p^{s}\right)^{\frac{1}{s}} b, y\right) p^{s-1}\left(1-p^{s}\right)^{\frac{1-s}{s}} d p \leq f(a, y)+f(b, y) .
\end{gathered}
$$

Changing the notation $p=t$, the inequality (3.5) becomes

$$
\int_{0}^{1} f\left(t a+\left(1-t^{s}\right)^{\frac{1}{s}} b, y\right)\left[1+t^{s-1}\left(1-t^{s}\right)^{\frac{1-s}{s}}\right] d t \leq f(a, y)+f(b, y) .
$$

Since $y \in[c, d]$ then there is $\tau \in[0,1]$ such that $y=\tau c+(1-\tau) d$. Using the Breckner type convexity of $f$ with respect to the second co-ordinate in the right side of this inequality one gets

$$
\begin{gathered}
\int_{0}^{1} f\left(t a+\left(1-t^{s}\right)^{\frac{1}{s}} b, \tau c+(1-\tau) d\right)\left[1+t^{s-1}\left(1-t^{s}\right)^{\frac{1-s}{s}}\right] d t \\
\leq \tau^{\sigma}[f(a, c)+f(b, c)]+(1-\tau)^{\sigma}[f(a, d)+f(b, d)] .
\end{gathered}
$$

Changing the significance of $\tau$ into $1-\tau$, so $y=(1-\tau) c+\tau d$, one obtains a similar inequality

$$
\begin{gathered}
\left.\int_{0}^{1} f\left(t a+\left(1-t^{s}\right)^{\frac{1}{s}} b,(1-\tau) c+\tau\right) d\right)\left[1+t^{s-1}\left(1-t^{s}\right)^{\frac{1-s}{s}}\right] d t \\
\leq(1-\tau)^{\sigma}[f(a, c)+f(b, c)]+\tau^{\sigma}[f(a, d)+f(b, d)] .
\end{gathered}
$$

Compute the sum of (3.6) and (3.7) and integrate the resulted inequality with respect to $\tau$ over $[0,1]$, obtaining the required inequality. 
Theorem 3.2. Let two numbers $s, \sigma \in(0,1]$, not necessarily distinct, be fixed. Let $f:[0,+\infty)^{2} \rightarrow \mathbb{R}$ be a function that is (Orlicz, Breckner) coordinated $(s, \sigma)$-convex and $f \in L_{1}(D)$ on each rectangle $D=[a, b] \times[c, d] \subseteq$ $[0,+\infty)$. Then

$$
\begin{gathered}
\int_{c}^{d} \int_{0}^{1} f\left(t^{\frac{1}{s}} a+(1-t)^{\frac{1}{s}} b, y\right) d t d y \\
\leq \frac{d-c}{2(\sigma+1)}[f(a, c)+f(a, d)+f(b, c)+f(b, d)] .
\end{gathered}
$$

Proof. First we use the $s$-convexity in the first sense of function $f$ with respect to the first coordinate. Taking $t \in[0,1]$ and $y \in[c, d]$ one gets the following inequalities:

$$
\begin{aligned}
& f\left(t^{\frac{1}{s}} a+(1-t)^{\frac{1}{s}} b, y\right) \leq t f(a, y)+(1-t) f(b, y) \\
& f\left((1-t)^{\frac{1}{s}} a+t^{\frac{1}{s}} b, y\right) \leq(1-t) f(a, y)+t f(b, y)
\end{aligned}
$$

Computing the sum of (3.9) and (3.10) one gets

$$
f\left(t^{\frac{1}{s}} a+(1-t)^{\frac{1}{s}} b, y\right)+f\left((1-t)^{\frac{1}{s}} a+t^{\frac{1}{s}} b, y\right) \leq f(a, y)+f(b, y) .
$$

Integrate this inequality with respect to $t$ over $[0,1]$.

$$
\begin{gathered}
\int_{0}^{1} f\left(t^{\frac{1}{s}} a+(1-t)^{\frac{1}{s}} b, y\right) d t+\int_{0}^{1} f\left((1-t)^{\frac{1}{s}} a+t^{\frac{1}{s}} b, y\right) d t \\
\leq f(a, y)+f(b, y)
\end{gathered}
$$

which is equivalent to

$$
2 \int_{0}^{1} f\left(t^{\frac{1}{s}} a+(1-t)^{\frac{1}{s}} b, y\right) d t \leq f(a, y)+f(b, y) .
$$

Since $y \in[c, d]$ then $y=\tau c+(1-\tau) d$ for some $\tau \in[0,1]$. By the Breckner type convexity of $f$ with respect to the second co-ordinate (3.12) becomes

$$
\begin{gathered}
2 \int_{0}^{1} f\left(t^{\frac{1}{s}} a+(1-t)^{\frac{1}{s}} b, \tau c+(1-\tau) d\right) d t \\
\leq \tau^{\sigma} f(a, c)+(1-\tau)^{\sigma} f(a, d)+\tau^{\sigma} f(b, c)+(1-\tau)^{\sigma} f(b, d) .
\end{gathered}
$$

Putting $y=(1-\tau) c+\tau d, \tau \in[0,1]$, one derives:

$$
2 \int_{0}^{1} f\left(t^{\frac{1}{s}} a+(1-t)^{\frac{1}{s}} b,(1-\tau) c+\tau d\right) d t
$$




$$
\leq(1-\tau)^{\sigma} f(a, c)+\tau^{\sigma} f(a, d)+(1-\tau)^{\sigma} f(b, c)+\tau^{\sigma} f(b, d) .
$$

The sum of (3.13) and (3.14) can be written as

$$
\begin{gathered}
4 \int_{0}^{1} f\left(t^{\frac{1}{s}} a+(1-t)^{\frac{1}{s}} b, \tau c+(1-\tau) d\right) d t \\
\leq\left[\tau^{\sigma}+(1-\tau)^{\sigma}\right][f(a, c)+f(a, d)+f(b, c)+f(b, d)] .
\end{gathered}
$$

Integrating this inequality with respect to $\tau$ over $[0,1]$ one obtains the required inequality.

Remark 3.1. If $s=\sigma=1$ then both inequalities (2.2) and (2.4) reduce to the upper bound from [11], Theorem 1.

Remark 3.2. Inequalities (3.8) and (3.8) are sharp. We prove their sharpness as in the case of the lower bounds. Let us chose function $f$ to be Orlicz-affine in the first co-ordinate and Breckner-affine in the second coordinate, which means that $f(x, y)=k$ constant, according to [17]. Let us take $s=\sigma=1$. Then inequalities become $(d-c) k \leq(d-c) k$ in this case, which proves their sharpness.

We have considered two variables co-ordinated generalized convex functions obtained by means of the $s$-convexity both in the first and in the second sense. Combining the two kinds of $s$-convexity in all possible manners we obtained three concepts of co-ordinated $(s, \sigma)$-convexity. Some of these convexities were previously studied in the mathematical inequalities literature. One of these concepts, called (Orlicz, Breckner) co-ordinated $(s, \sigma)$-convexity, is new. Several new Hermite-Hadamard type integral inequalities for (Orlicz, Breckner) co-ordinated $(s, \sigma)$-convex functions are established. Results proved in this paper continue to hold for all the three kinds of co-ordinated $(s, \sigma)$-convex functions, when one of them intersects the (Orlicz, Breckner) co-ordinated $(s, \sigma)$-convexity. The sharpness of these new inequalities is proved. The ideas and techniques of this paper may be useful in obtaining integral inequalities for other classes of two variables functions having generalized convexities on co-ordinates. This is an interesting problem of future research.

\section{References}

[1] A.O. Akdemir and M.E. Özdemir, Some Hadamard-type inequalities for coordinated $p$-convex functions and Godunova-Levin functions, arXiv:1012.5880v2 [math.CA], (2010) 
[2] M. Alomari and M. Darus, Co-ordinated $s$-convex functions in the first sense with some Hadamard-type inequalities, Int. J. Contemp. Math. Sci., 3, (2008), 1557-1567

[3] M. Alomari and M. Darus, The Hadamard's inequality for $s$-convex function of 2-variables on the co-ordinates, Int. J. Math. Anal., 13, (2008), 629-638

[4] S.P. Bai and F. Qi, Some inequalities for $\left(s_{1}, m_{1}\right)-\left(s_{2}, m_{2}\right)$-convex functions on the co-ordinates, Global J. Math. Anal., 1, (2013), 22-28

[5] W.W. Breckner, Stetigkeitsaussagen für eine Klasse verallgemeinerter konvexer funktionen in topologischen linearen Räumen, Publ. Inst. Math., 23, (1978), 13-20

[6] W.W. Breckner and B. Orbán, Continuity properties of rationally s-convex mappings with values in an ordered topological linear space, Babeş-Bolyai University of Cluj-Napoca Publishing House, Cluj-Napoca, 1978

[7] G. Cristescu, M.A. Noor, and M.U. Awan, Bounds of the second degree cumulative frontier gaps of functions with generalized convexity, Carpathian J. Math., 31, (2015), 173-180

[8] G. Cristescu, M.A. Noor, K.I. Noor, and M.U. Awan, Some Inequalities for functions having Orlicz-convexity, Appl. Math. Comp, 273, (2016), 226-236

[9] S.S. Dragomir and S. Fitzpatrick, The Hadamard's inequality for s-convex functions in the second sense, Demonstratio Math., 32, (1999), 687-696

[10] S.S. Dragomir and C.E.M. Pearce, Selected topics on Hermite-Hadamard inequalities and applications, 2000

[11] S.S. Dragomir, On Hadamard's inequality for convex functions on the co-ordinates in a rectangle from the plane, Taiwanese J. Math., 5, (2001), 775-788

[12] J. Hadamard, Étude sur les propriétés des fonctions entières et en particulier d'une fonction considerée par Riemann, J. Math Pures Appl., 58, (1893), 171-215

[13] Ch. Hermite, Sur deux limites d'une intégrale définie, Mathesis, 3, (1883)

[14] H. Hudzik and L. Maligranda, Some remarks on s-convex functions, Aequationes Math., 48, (1994), 100-111

[15] J.L.W.V. Jensen, Sur les fonctions convexes et les inégalités entre les valeurs moyennes, Acta. Math., 30, (1906), 175-193

[16] M.A. Latif, Some new Hermite-Hadamard type inequalities for functions whose higher order partial derivatives are co-ordinated s-convex, Kragujevac J. Math., 38, (2014), 125-146

[17] G. Maksa and Z. Páles, The equality case in some recent convexity inequalities, Opuscula Math., 31, (2011), 269-277

[18] M. Matłoka, On some Hadamard-type inequalities for $\left(h_{1}, h_{2}\right)$-preinvex functions on the co-ordinates, J. Inequal. Appl., (2013), 2013:27

[19] M.A. Noor, G. Cristescu, and M.U. Awan, Generalized fractional HermiteHadamard inequalities for twice differentiable $s$-convex functions, Filomat, 29, (2015), $807-815$

[20] W. Orlicz, A note on modular spaces I, Bull. Acad. Polon. Sci. Math. Astronom. Phys., 9, (1961), 157-162 
[21] M.E. Özdemir, H. Kavurmaci, A.O. Akdemir, and M. Avci, Inequalities for convex and $s$-convex functions on $\Delta=[a, b] \times[c, d], J$. Inequal. Appl., (2012), 2012:20

[22] J. Park, On the Hermite-Hadamard-like type inequalities for co-ordinated $(s, r)$ convex mappings in the first sense, Int. J. Pure Appl. Math., 74, (2012), 251-263

[23] M.Z. Sarikaya, E. Set, M.E. Özdemir, and S.S. Dragomir, New some Hadamard's type inequalities for co-ordinated convex functions, Tamsui Oxford J. Math. Sci., 28, (2011)

[24] B-Y. Xi, J. Hua, and F. Qi, Hermite-Hadamard type inequalities for extended $s$-convex functions on the co-ordinates in a rectangle, Journal of Applied Analysis, 20, (2014), 1-17

Gabriela Cristescu

Department of Mathematics and Computer Sciences

Aurel Vlaicu University of Arad

Bd. Revoluţiei nr.77, Arad

România

E-mail: gabriela.cristescu@uav.ro

Muhammad Aslam Noor

Department of Mathematics

COMSATS Institute of Information Technology

Park Road, Islamabad, Pakistan

E-mail: noormaslam@hotmail.com

Muhammad Uzair Awan

Government College University

Faisalabad, Pakistan

E-mail: awan.uzair87@yahoo.com

Mihail Găianu

Department of Computer Sciences

West University of Timişoara

Vasile Pârvan nr. 4, Timişoara

România

E-mail: mgaianu@info.uvt.ro

Received: 23.09.2015

Accepted: 27.12.2015 